\title{
Mathematical Reasoning Ability Through Interpretation-construction Design Model
}

\author{
Rafiq Zulkarnaen \\ Department of Mathematics Education, \\ University of Singaperbangsa Karawang \\ Karawang, Indonesia \\ Rafiq.zulkarnaen@staff.unsika.ac.id
}

\begin{abstract}
- the aim of this research is to examine the impact of students' ability on mathematical reasoning after they received learning treatment of Interpretation-construction design model (IC). Quasi-experimental methods were used in this study with non-equivalent pretest-posttest control group design. The population of the study involved 134 students of class $X$ in two schools in Bogor, West Java, Indonesia, with purposive sampling as the technique of collecting data. The instrument used was descriptive question of mathematical reasoning ability covering some aspects: analysis, synthesis, conjecturing, generalization, and justify. The result of this research concluded that interpretationconstruction design model impacts student mathematical reasoning ability. Reviewing from the students' answer descriptions, students' ability is still weak in the aspect synthesis and draw-conclution.
\end{abstract}

Keywords-contextualization; cognitive apprenticeship; reasoning; colaboration.

\section{INTRODUCTION}

Most mathematics curricula recognize that reasoning are fundamental aspects of mathematics learning [1]. Students must have mathematical reasoning abilities when learning mathematics. In learning mathematics, students must learn not only memorize rules and procedures presented by teachers or textbooks but learning mathematics should make sense and be logical [2] to develop an understanding of situations, contexts, or concepts by linking existing knowledge, and making a statement. Making sense is part of mathematical reasoning. Reasoning involves conclusions drawn from principle or from evidence, and reasoning is making an argument for explaining a fact [3].

Communication is an integral part of the process of reasoning, both for an individual working with previously produced texts to produce a new one, and for groups working together to produce an argument. The texts or products of reasoning have, as their main purpose, to communicate reasoning [4]; real life contexts give students the possibility to connect familiar situations and infer their mathematical reasoning [5]. Learning models that include student activities in observing and solving real-world problems, collaborating, contextualizing are interpretation-construction design model (ICON-model). The ICON model emphasizes students' interpretations of information and their processes of knowledge construction [6]. Principles of the ICON model: observations in authentic activities, interpretation construction, contextualization, cognitive apprenticeship, colaboration, multiple interpretations and manifestation [6], [7]

The aim of this study is therefore to examine the efficacy or impact of ICON-model on students' mathematical reasoning ability.

\section{LITERATURE REVIEW}

Authentic learning experiences or connecting the real world and mathematics learning should be taken into students activities. Authentic observation and experience allow students to incorporate concepts and theories to apply them in real-world context. authentic learning has been far less theorized and applied in social science disciplines, which require students to not only understand the content matter of the discipline and its possible application, but also to be critical of the discipline and its wider contexts [8]. If there are no authentic observations, students will learn through rote memorization and they cannot use it in an appropriate context [7].

Contextualization occurs when students access the prior knowledge of various materials to aid interpretation and argumentation [6]. Contextualizing a problem can mean connecting a problem with a particular physical situation, but it can also connect problems with other ideas. Three specific contexts to analyze for mathematical understanding: The situational context refers to the interaction of the individual with the materials, environment, sensations, and actions involved; The cultural context refers to discursive rules, conventions, and patterns of behavior; The conceptual context is involved with personal constructions of concepts of the situation[9].

Cognitive apprenticeship means combining the core technology of traditional apprenticeship with school education to cultivate the students' cognitive skills, namely, the ability needed by the experts to think, to solve problems and to deal with sophisticated tasks [10]. Cognitive apprenticeship concists of: modeling, coaching, scaffolding, articulation, reflection, and exploration [11]. Vygotsky's theory of Cognitive Development introduced the possibility of accounting for individual cognition and difference as informed by social activity, and development always follows the student's potential to learn [12]. A social activity in which 
students are engaged in meaning construction through discussion, argumentation and negotiation among teachers, peers and other students[7]. The language that teachers use in explaining mathematical concepts to students is a crucial factor in the success or failure of student learning [13]

\section{RESEARCH METHOD}

This study was conducted in equation and quadratic function lesson at one public high school in Bogor West Java Indonesia during the first semester of 2013. Out of 340 students of nine classes enrolled, sixty eight volunteered to begin the study. These students participated in lesson equation and quadratic function material using interpretation-construction learning design model, and mathematical reasoning test that was approximately 2.5 months long. Because such designs do not involve random assignment of participants to conditions, quasiexperimental with pretest-posttest control group design used in this research. From one school selected, two classes were taken for experimental and control class with 34 students each.

\section{A. Instrument}

To measure students mathematical reasoning abilities, five mathematical reasoning problems are used, with indicators of mathematical reasoning capabilities adopted from [14] view of students' abilities in: Analyze (Determine, describe, or use relationships among numbers, expressions, quantities, and shapes); Synthesize (Link different elements of knowledge, related representations, and procedures to solve problems); Evaluate (Evaluate alternative problem solving strategies and solutions); Draw Conclusion (Make valid inferences on the basis of information and evidence); Generalize (Make statements that represent relationships in more general and more widely applicable terms); Justify (Provide mathematical arguments to support a strategy or solution).

\section{B. Method of data analysis}

Quantitative data analysis is needed to prove the hypothesis, with data analysis technique done through several stages. The first stage, testing the statistical test requirements used whether data processing using parametric or non parametric statistics as the basis in testing the hypothesis. Furthermore, test of difference of pretest and posttest data to know which learning model give influence significantly to mathematical reasoning ability.

\section{RESUlT AND DisCUSSION}

Data of pretest and postest score of mathematical reasoning ability are described and analyzed based on learning treatment factor (ICON-model and Conventional Learning) and prior knowledge test category (high, medium, and low). As an overview, the scores (minimum, maximum, average, and standard deviations) are presented based on each of the factors presented in Table 1.
TABLE I. PRETEST SCORE OF MATHEMATICAL REASONING ABILITY

\begin{tabular}{|c|c|c|c|c|c|}
\hline \multirow{2}{*}{ Treatment } & \multirow{2}{*}{$\begin{array}{c}\text { MPA } \\
\text { category }\end{array}$} & \multicolumn{2}{|c|}{ Pretest } & \multicolumn{2}{c|}{ Postest } \\
\cline { 2 - 6 } & High & 3.83 & 1.22 & 17.67 & 1.22 \\
\hline \multirow{4}{*}{ ICON } & Medium & 3.07 & 1.68 & 14.52 & 2.13 \\
\cline { 2 - 6 } & Low & 3.22 & 1.34 & 13.00 & 1.21 \\
\cline { 2 - 6 } & Total & $\mathbf{3 . 1 1}$ & $\mathbf{1 . 0 2}$ & $\mathbf{1 4 . 7 6}$ & $\mathbf{1 . 2 2}$ \\
\hline \multirow{4}{*}{ Conventional } & High & 3.67 & 1.34 & 14.67 & 3.11 \\
\cline { 2 - 6 } & Medium & 3.39 & 1.34 & 12.65 & 1.22 \\
\cline { 2 - 6 } & Low & 3.01 & 1.45 & 13.20 & 2.45 \\
\cline { 2 - 6 } & Total & $\mathbf{3 . 1 2}$ & $\mathbf{1 . 2 3}$ & $\mathbf{1 3 . 0 9}$ & $\mathbf{2 . 5 6}$ \\
\hline
\end{tabular}

As indicated in Table 1, there is no significant difference of students' mathematical reasoning ability before treatment is given to each sample group, based on achievement of mathematical prior ability (MPA), and learning design model. Having given the learning treatment of the interpretationconstruction and conventional learning to each sample group, posttest data indicate that there is a difference in the ability of mathematical reasoning as presented in Figure 1.

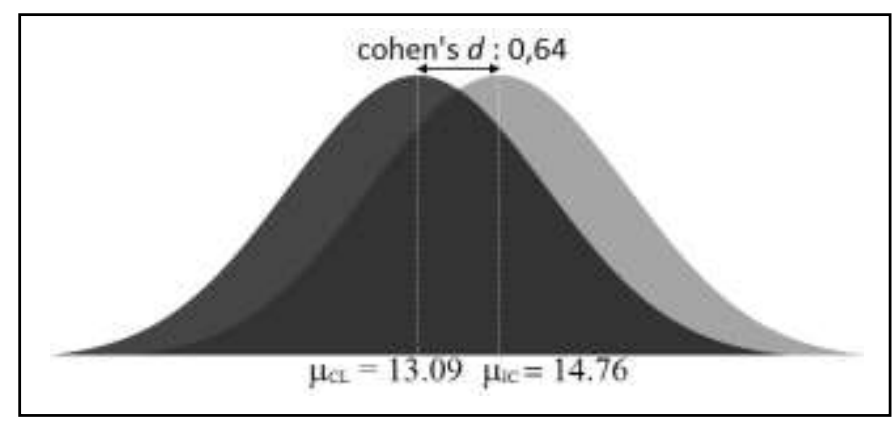

Fig. 1. Cohen's calculation postest

Based on Figure 1, with a Cohen's $d$ of $0.64,74 \%$ of the treatment group will be above the mean of the control group (Cohen's $\mathrm{U}_{3}$ ), $75 \%$ of the two groups will overlap, and there is a $67 \%$ chance that a person picked at randomly from the treatment group will have a higher score than a person picked at randomly from the control group.

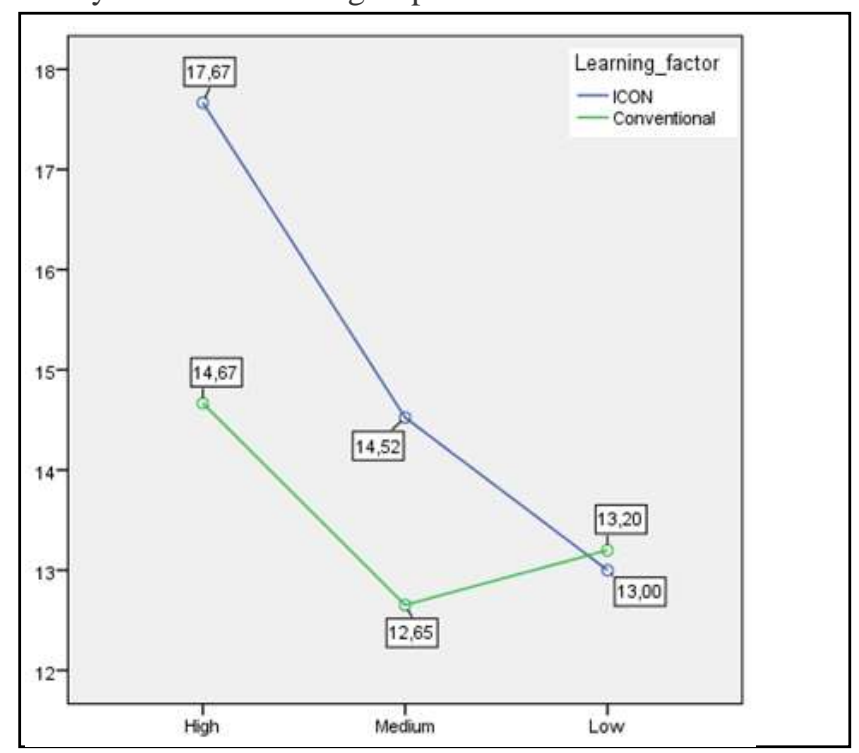

Fig.2. Interaction between learning model and MPA 
Teachers play an important role in building students' mathematical reasoning abilities. Classroom management and support for students during the mathematics learning is very important. Learning mathematics using ICON-models, whether directly or indirectly, plays a very important role. In the authentic observation phase, the teacher presents real-world problems or links the knowledge that students learn in the classroom and its relevance to the real world. This can be done by the teacher through explaining to the students what they will learn and making the lesson meaningful to the students.

\section{REFERENCES}

[1] G. Hanna, "Mathematical Proof, Argumentation, and Reasoning," in Encyclopedia of mathematics education, S. Lerman, Ed. New York: SpringerReference, 2014, p. 423.

[2] E. Ruhama and Z. Markovits, "Some aspects of teachers' and students' views on student reasoning and knowledge construction," Int. J. Math. Educ. Sci. Technol., vol. 26, no. 4, pp. 531-544, 1995.

[3] G. Kosyvas, "Levels of arithmetic reasoning in solving an open-ended problem,” Int. J. Math. Educ. Sci. Technol., vol. 47, no. 3, pp. 356-372, 2016.

[4] K. Brodie, Teaching mathematical reasoning in secondary school classrooms. New York: Springer, 2010.

[5] M. Schindler and S. Hußmann, "Sixth-grade students' reasoning on the order relation of integers as influenced by prior experience: an inferentialist analysis," Math. Educ. Res. J., vol. 29, no. 4, pp. 471-492, 2017.

[6] J. B. Black and R. O. Mcclintock, "An Interpretation Construction Approach to Constructivist Design," in Constructivist learning environments, B. Wilson, Ed. Englewood Cliffs, NJ: Educational Technology Publications, 1995, pp. 25-31.

Fig.3. mathematical reasoning achievement

Based on figure 3 above, students are still weak in the aspect of synthesize and draw conclution, and the biggest aspect in mathematical reasoning is evaluate and analyze. This may be caused by ICON learning process begins with authentic observation; through observation the students are asked to mathematize and demathematize real world problem, so learning activities enable students to be proficient in the aspects of evaluate and analize.

The result of this research show that cognitive apprenticeship step of ICON-model support the students' thinking, form modeling, scaffolding and also colaboration. Interventions on collaborative working can improve communication among students since they the quality of the students' communication and thus influence students' mathematical reasoning. Teacher also has an important role in modeling. They use language to help students make connections between their own images and the concepts they meet within the language of mathematics [13].

Learning mathematics using ICON-model focuses more on conceptual understanding, and conceptual understanding is capable of giving effect to procedural fluency. Futhermore, prompting students by posing prediction questions at the launch of a lesson engaged all students in the classroom. Thus, prediction questions provided an opportunity for all students to build upon their conceptual understanding and reasoning of mathematical ideas [15] .
[7] C. Tsai, "The interpretation construction design model for teaching science and its applications to Internet-based instruction in Taiwan," Int. J. Educ. Dev., vol. 21, no. 5, pp. 401-415, 2001.

[8] F. Borthwick, S. Bennett, G. Lefoe, and E. Huber, "Applying authentic learning to social science: A learning design for an inter-disciplinary sociology subject," J. Learn. Des., vol. 2, no. 1, pp. 14-24, 2007.

[9] S. P. Yee and J. D. Bostic, "Developing a contextualization of students ' mathematical problem solving," J. Math. Behav., vol. 36, pp. 1-19, 2014.

[10] G. Shan, "Cognitive Apprenticeship --- An Effective Learning Mode In the College English Listening Teaching," Asian Soc. Sci., vol. 4, no. 7, pp. $157-158,2008$.

[11] A. Collin and J. S. Brown, "Cognitive apprenticeship: Teaching the craft of reading, writing, and mathematics," in Knowing, learning, and instruction: Essays in honor of Robert Glaser, L. B. Resnick, Ed. Hillsdale, NJ: Lawrence Erlbaum Associates, 1989, pp. 453-494.

[12] M. Walshaw, "Understanding mathematical development through Vygotsky," Res. Math. Educ., vol. 19, no. 3, pp. 293-309, 2017.

[13] M. Y. Lai, "Constructing Meanings of Mathematical Registers Using Metaphorical Reasoning and Models," Math. Teach. Educ. Dev., vol. 15, no. 1 , pp. 29-47, 2013

[14] I. V. S. Mullis and M. O. Martin, TIMSS 2015Assessment Frameworks. Boston, Chesnut Hill: TIMSS \& PIRLS International Study Center, Association for the Evaluation of Educational Achievement (IEA), 2013.

[15] L. Kasmer and O. Kim, "Using Prediction to Promote Mathematical Understanding and Reasoning," Using Predict. to Promot. Math. Underst. Reason., vol. 111, no. 1, pp. 20-33, 2006. Lynch School of Education, Boston College and International 